A N N A LES

UNIVERSITATIS MARIAE CURIE-SKŁODOWSKA

L UBLIN - POLONIA

VOL. LXIX, NO. 2, 2015

SECTIO A

$73-83$

\author{
AGNIESZKA TANAŚ
}

\title{
A continuum individual based model of fragmentation: dynamics of correlation functions
}

\begin{abstract}
An individual-based model of an infinite system of point particles in $\mathbb{R}^{d}$ is proposed and studied. In this model, each particle at random produces a finite number of new particles and disappears afterwards. The phase space for this model is the set $\Gamma$ of all locally finite subsets of $\mathbb{R}^{d}$. The system's states are probability measures on $\Gamma$ the Markov evolution of which is described in terms of their correlation functions in a scale of Banach spaces. The existence and uniqueness of solutions of the corresponding evolution equation are proved.
\end{abstract}

1. Introduction. Mathematical models describing large ecological communities mostly operate with averaged quantities like the density of the entities constituting the community and are deduced in a phenomenological way, see, e.g. $[3,15,17]$. In a more advanced modeling, the dynamical acts of each single entity are being taken into account. Among such individualbased models one might distinguish those where the entities disappear (die) or give birth to new ones, see, e.g. $[5,7,9]$. In the present paper, we introduce and study an individual-based model of an infinite system of point 'particles' placed in $\mathbb{R}^{d}$, in which each 'particle' produces at random a finite 'cloud' (possibly empty) of new ones, and disappears afterwards. A particular case of this model with the cloud being empty or consisting of exactly

2010 Mathematics Subject Classification. 60J80, 82C21, 92D25.

Key words and phrases. Configuration space, individual-based model, birth-and-death process, correlation function, scale of Banach spaces, Ovcyannikov method. 
two offsprings can describe the dynamics of cell division. As is now commonly adopted, see [16], the phase space of such systems is the configuration space $\Gamma=\Gamma\left(\mathbb{R}^{d}\right)$ which consists of all locally finite subsets of $\mathbb{R}^{d}$, called configurations. This set is endowed with a measurability structure that allows one to employ probability measures defined thereon. Such measures are then considered as the system's states the Markov evolution of which is described by means of the corresponding Fokker-Planck equation. Its dual is the Kolmogorov equation involving observables - appropriate functions $F: \Gamma \rightarrow \mathbb{R}$. Details of the analysis on configuration spaces can be found in $[1,10,12]$, see also $[4,5,6,7]$ for more on individual-based modeling of continuum infinite-particle systems. In studying the model proposed in this work we follow the so-called statistical approach in which the evolution of states is described as the evolution of the corresponding correlation functions. It is obtained by solving the evolution equation deduced from the Fokker-Planck equation by means of a certain procedure, see [8]. In this paper, we prove the existence and uniqueness of the classical solutions of this evolution equation. It has been done by means of an Ovcyannikov-type method, see [4, 18], in a scale of Banach spaces of correlation functions. As typical of this method, the solution is shown to exist only on bounded time interval.

2. Preliminaries. The configuration space $\Gamma$ over $\mathbb{R}^{d}$ is defined as

$$
\Gamma=\left\{\gamma \subset \mathbb{R}^{d}:|\gamma \cap K|<\infty \text { for any compact } K \subset \mathbb{R}^{d}\right\},
$$

where $|\cdot|$ stands for cardinality. It is equipped with the weakest topology for which the mappings

$$
\Gamma \ni \gamma \mapsto \sum_{x \in \gamma} f(x),
$$

are continuous for all continuous compactly supported functions $f: \mathbb{R}^{d} \rightarrow \mathbb{R}$. This topology can be metrized in the way that makes $\Gamma$ a Polish space. We denote by $\mathcal{B}(\Gamma)$ the corresponding Borel $\sigma$-field on $\Gamma$. The system's states are probability measures on $(\Gamma, \mathcal{B}(\Gamma))$ the set of which is denoted by $\mathcal{P}(\Gamma)$. Note that the points of $\Gamma$ can be associated with elements of $\mathcal{P}(\Gamma)$ by assigning the corresponding Dirac measures $\gamma \mapsto \delta_{\gamma} \in \mathcal{P}(\Gamma)$. Such elements of $\Gamma$ are called point states. The evolution of the states of a given system is described by the Fokker-Planck equation

$$
\frac{d}{d t} \mu_{t}=L^{*} \mu_{t},\left.\quad \mu_{t}\right|_{t=0}=\mu_{0}, \quad t>0,
$$

in which the 'operator' $L^{*}$ contains the whole information about the system. Along with states $\mu \in \mathcal{P}(\Gamma)$ one can also consider suitable functions $F: \Gamma \rightarrow$ $\mathbb{R}$, called observables. Then the number

$$
\int_{\Gamma} F d \mu
$$


is the value of $F$ in state $\mu$. In particular, $F(\gamma)$ is the value of $F$ in the point state $\gamma$. The evolutions of states and observables are related to each other by the duality

$$
\int_{\Gamma} F_{0} d \mu_{t}=\int_{\Gamma} F_{t} d \mu_{0}, \quad t>0 .
$$

Hence, the system's evolution can also be considered as the evolution of observables, obtained from the Kolmogorov equation

$$
\frac{d}{d t} F_{t}=L F_{t},\left.\quad F_{t}\right|_{t=0}=F_{0}, \quad t>0
$$

dual in the sense of (2.2) to that in (2.1). For the most of such models, also for that introduced and studied in this work, the direct solving of (2.1) is possible only for finite systems, i.e., in the case where the states are supported on the subset of $\Gamma$ consisting of finite configurations only, see, e.g. [14]. As we are going to describe infinite systems, we will follow another approach based on the use of correlation functions.

The space of finite configurations mentioned above can be given by writing it as the topological sum

$$
\Gamma_{0}:=\bigsqcup_{n=0}^{\infty} \Gamma^{(n)},
$$

where

$$
\Gamma^{(0)}=\{\emptyset\}, \quad \Gamma^{(n)}:=\left\{\eta \subset \mathbb{R}^{d}:|\eta|=n\right\}, \quad n \in \mathbb{N} .
$$

Here each $\Gamma^{(n)}$ is equipped with the topology related to the Euclidean topology of the underlying space $\mathbb{R}^{d}$. One can show that $\Gamma_{0} \in \mathcal{B}(\Gamma)$ and that the corresponding Borel $\sigma$-field of subsets of $\Gamma_{0}$ coincides with the $\sigma$-field

$$
\mathcal{B}\left(\Gamma_{0}\right)=\left\{A \cap \Gamma_{0}: A \in \mathcal{B}(\Gamma)\right\} .
$$

Furthermore, a function $G: \Gamma_{0} \rightarrow \mathbb{R}$ is $\mathcal{B}\left(\Gamma_{0}\right)$-measurable if and only if there exists a family $\left\{G^{(n)}\right\}_{n \in \mathbb{N}_{0}}$ such that: (a) $G^{(0)}$ is just a real number; (b) $G^{(1)}: \mathbb{R}^{d} \rightarrow \mathbb{R}$ is a Borel function; (c) for each integer $n \geq 2, G^{(n)}$ : $\left(\mathbb{R}^{d}\right)^{n} \rightarrow \mathbb{R}$ is a symmetric Borel function; (d) $G^{(0)}=G(\emptyset)$ and for each $n \in \mathbb{N}$, the following holds

$$
G^{(n)}\left(x_{1}, \ldots, x_{n}\right)=G\left(\left\{x_{1}, \ldots, x_{n}\right\}\right) .
$$

Note that, for $G$ as above, the family of Borel functions $\left\{G^{(n)}\right\}_{n \in \mathbb{N}_{0}}$ is not unique. Let $B_{\text {loc }}\left(\Gamma_{0}\right)$ stand for the set of all functions $G: \Gamma_{0} \rightarrow \mathbb{R}$ for each of which there exists the family as mentioned above with the following properties: (a) each $G^{(n)}, n \in \mathbb{N}$, is continuous and compactly supported; 
(b) there exists $N \in \mathbb{N}_{0}$ such that $G^{(n)} \equiv 0$ for all $n \geq N$. The LebesguePoisson measure $\lambda$ on $\Gamma_{0}$ is defined by the following integrals

$$
\int_{\Gamma_{0}} G(\eta) \lambda(d \eta)=G^{(0)}+\sum_{n=1}^{\infty} \frac{1}{n !} \int_{\left(\mathbb{R}^{d}\right)^{n}} G^{(n)}\left(x_{1}, \ldots, x_{n}\right) d x_{1} \cdots d x_{n}
$$

where $G$ runs through $B_{\text {loc }}\left(\Gamma_{0}\right)$.

As mentioned above, the problem (2.1) will be solved in terms of correlation functions. To introduce them we use Bogoliubov functionals, see [2]. Let $\Theta$ be the set of all continuous compactly supported functions $\theta: \mathbb{R}^{d} \rightarrow(-1,0]$. For a given $\mu \in \mathcal{P}(\Gamma)$, the Bogoliubov functional is

$$
B_{\mu}(\theta)=\int_{\Gamma} \prod_{x \in \gamma}(1+\theta(x)) \mu(d \gamma), \quad \theta \in \Theta
$$

The integral in (2.5) makes sense for each $\theta \in \Theta$ as the map

$$
\gamma \mapsto \prod_{x \in \gamma}(1+\theta(x))
$$

is measurable and bounded. The key idea of the approach which we follow in this work is to restrict the choice of $\mu_{0}$ in $(2.1)$ to the subset of $\mathcal{P}(\Gamma)$ consisting of all the states $\mu$ with the property: $B_{\mu}$ can be continued to a function of $\theta \in L^{1}\left(\mathbb{R}^{d}\right)$ analytic in some neighborhood of the point $\theta=$ 0 . This exactly means, see $[8,11]$, that $(2.5)$ can be written down in the following form

$$
\begin{aligned}
B_{\mu}(\theta) & =\int_{\Gamma_{0}}\left(k_{\mu}(\eta) \prod_{x \in \eta} \theta(x)\right) \lambda(d \eta) \\
& =1+\sum_{n=1}^{\infty} \frac{1}{n !} \int_{\left(\mathbb{R}^{d}\right)^{n}} k_{\mu}^{(n)}\left(x_{1}, \ldots, k_{n}\right) \prod_{i=1}^{n} \theta\left(x_{i}\right) d x_{1} \cdots d x_{n},
\end{aligned}
$$

where $\lambda$ is as in (2.4) and $k_{\mu}$ (resp. $k_{\mu}^{(n)}$ ) is the correlation function (resp. $n$-th order correlation function) of the state $\mu$ such that $k_{\mu}^{(n)} \in L^{\infty}\left(\left(\mathbb{R}^{d}\right)^{n}\right)$ for all $n \in \mathbb{N}$. Note that in this case $k_{\mu}$ and $\mu$ are related to each other by

$$
\int_{\Gamma}\left(\sum_{\eta \subset \gamma} G(\eta)\right) \mu(d \gamma)=\int_{\Gamma_{0}} G(\eta) k_{\mu}(\eta) \lambda(d \eta),
$$

holding for all $G \in B_{\text {loc }}\left(\Gamma_{0}\right)$. By means of (2.6) one can transfer the action of $L$ as in (2.3) from $F_{\theta}(\gamma):=\prod_{x \in \gamma}(1+\theta(x))$ to $k_{\mu}$ according to the following rule, cf. (2.2) and (2.7),

$$
\int_{\Gamma}\left(L F_{\theta}\right)(\gamma) \mu(d \gamma)=\int_{\Gamma_{0}}\left(L^{\Delta} k_{\mu}\right)(\eta) \prod_{x \in \eta} \theta(x) \lambda(d \eta)
$$


This leads one from the Kolmogorov equation (2.3) to the problem

$$
\frac{d}{d t} k_{t}=L^{\Delta} k_{t},\left.\quad k_{t}\right|_{t=0}=k_{\mu_{0}},
$$

which we study in the next section. Among other methods we use the Minlos lemma in the following form

Lemma 2.1 (Lemma 2.4 [8]). For Lebesgue-Poisson measure $\lambda$ defined in (2.4) and for any measurable function $M: \Gamma_{0} \times \Gamma_{0} \times \Gamma_{0} \rightarrow \mathbb{R}_{+}$the following holds

$$
\int_{\Gamma_{0}}\left(\sum_{\zeta \subset \eta} M(\zeta, \eta, \eta \backslash \zeta)\right) \lambda(d \eta)=\int_{\Gamma_{0}} \int_{\Gamma_{0}} M(\zeta, \eta \cup \zeta, \eta) \lambda(d \zeta) \lambda(d \eta)
$$

if both sides are finite.

3. The model. The model which we introduce and study in this work is specified by the generator, see (2.3),

$$
(L F)(\gamma)=\sum_{x \in \gamma} \int_{\Gamma_{0}} b(x \mid \xi)[F(\gamma \backslash x \cup \xi)-F(\gamma)] \lambda(d \xi) .
$$

In (3.1), the kernel $b(x \mid \xi) \geq 0$ describes the following act: the point $x \in$ $\gamma$ disappears and a finite configuration (cloud) $\xi \in \Gamma_{0}$ appears instead. A particular case where $b(x \mid \emptyset)=m(x), b\left(x \mid\left\{y_{1}, y_{2}\right\}\right)=c\left(x \mid y_{1}, y_{2}\right)$, and $b(x \mid \xi) \equiv 0$ for other $\xi$, is a cell division model, cf. [3, 15], in which each cell can die with intrinsic mortality rate $m(x)$ or split, with rate $c\left(x \mid y_{1}, y_{2}\right)$, into two new cells located at $y_{1}$ and $y_{2}$. In this case $L$ takes the form

$$
\begin{aligned}
(L F)(\gamma)= & \sum_{x \in \gamma} m(x)[F(\gamma \backslash x)-F(\gamma)] \\
& +\int_{\mathbb{R}} \int_{\mathbb{R}} \sum_{x \in \gamma} c\left(x \mid y_{1}, y_{2}\right)\left[F\left(\gamma \backslash x \cup y_{1} \cup y_{2}\right)-F(\gamma)\right] .
\end{aligned}
$$

For

$$
c\left(x \mid y_{1}, y_{2}\right)=\frac{1}{2}\left(\delta\left(y_{1}-x\right) a^{+}\left(y_{2}-x\right)+\delta\left(y_{2}-x\right) a^{+}\left(y_{1}-x\right)\right)
$$

it turns into the contact model studied in [13].

By (2.8) and Lemma 2.1 we obtain from (3.1) the following

$$
\left(L^{\Delta} k\right)(\eta)=-E(\eta) k(\eta)+\int_{\mathbb{R}^{d}} \sum_{\zeta \subset \eta, \zeta \neq \emptyset} \beta(x \mid \zeta) k(\eta \cup x \backslash \zeta) d x,
$$

where

$$
\beta(x \mid \zeta):=\int_{\Gamma_{0}} b(x \mid \xi \cup \zeta) \lambda(d \xi), \quad E(\eta):=\sum_{x \in \eta} \beta(x \mid \emptyset)
$$


Regarding the kernel $b$ along with the standard measurability we assume that:

$$
\begin{array}{ll}
\exists \bar{\beta}>0 \quad \forall \eta \in \Gamma_{0} & \sum_{x \in \eta} b(x \mid \emptyset) \leq|\eta| \bar{\beta} ; \\
\exists \bar{\varphi}>0 \quad \forall \eta \in \Gamma_{0} \quad \int_{\mathbb{R}^{d}} \beta(x \mid \eta) d x=: \varphi(\eta) \leq \bar{\varphi} .
\end{array}
$$

Now we introduce the Banach spaces where we will solve the problem (2.9) with $L^{\Delta}$ given in (3.2). According to the assumption as to the Bogoliubov functional having the form (2.6) these are

$$
\mathcal{K}_{\alpha}=\left\{k: \Gamma_{0} \rightarrow \mathbb{R}:\|k\|_{\alpha}<\infty\right\}, \quad \alpha \in \mathbb{R},
$$

where

$$
\|k\|_{\alpha}=\sup _{n \in \mathbb{N}_{0}} \frac{1}{n !} e^{\alpha n}\left\|k^{(n)}\right\|_{L^{\infty}\left(\left(\mathbb{R}^{d}\right)^{n}\right)},
$$

which can also be written as

$$
\|k\|_{\alpha}=\operatorname{esssup}_{\eta \in \Gamma_{0}} \frac{1}{|\eta| !} e^{\alpha|\eta|}|k(\eta)| .
$$

Clearly,

$$
|k(\eta)| \leq|\eta| ! e^{-\alpha|\eta|}\|k\|_{\alpha}, \quad \eta \in \Gamma_{0} .
$$

In fact, we will consider the scale of such spaces $\left\{\mathcal{K}_{\alpha}: \alpha \in \mathbb{R}\right\}$. Naturally, $\|k\|_{\alpha^{\prime}} \geq\|k\|_{\alpha^{\prime \prime}}$ for $\alpha^{\prime}>\alpha^{\prime \prime}$; hence, $\mathcal{K}_{\alpha^{\prime}} \hookrightarrow \mathcal{K}_{\alpha^{\prime \prime}}$, that is, each smaller space is continuously embedded into each bigger one.

Let us write (3.2) in the form $L^{\Delta}:=A+B$ with

$$
\begin{gathered}
(A k)(\eta)=-E(\eta) k(\eta) \\
(B k)(\eta)=\int_{\mathbb{R}^{d}} \sum_{\zeta \subset \eta, \zeta \neq \emptyset} \beta(x \mid \zeta) k(\eta \cup x \backslash \zeta) d x .
\end{gathered}
$$

To define $L^{\Delta}$ as a linear operator in a given $\mathcal{K}_{\alpha}$ we set

$$
\mathcal{D}_{\alpha}(A)=\left\{k \in \mathcal{K}_{\alpha}: A k \in \mathcal{K}_{\alpha}\right\}
$$

and define $\mathcal{D}_{\alpha}(B)$ analogously. Then the domain of $L^{\Delta}$ in $\mathcal{K}_{\alpha}$ is set to be

$$
\mathcal{D}_{\alpha}\left(L^{\Delta}\right)=\mathcal{D}_{\alpha}(A) \cap \mathcal{D}_{\alpha}(B) .
$$

Let us prove that

$$
\forall \alpha^{\prime}>\alpha \quad \mathcal{K}_{\alpha^{\prime}} \subset \mathcal{D}_{\alpha}\left(L^{\Delta}\right) .
$$

By (3.3) and (3.5) we get from (3.6) the following estimates

$$
|(A k)(\eta)| \leq|\eta| \bar{\beta}|\eta| ! e^{-\alpha^{\prime}|\eta|}\|k\|_{\alpha^{\prime}}
$$




$$
\begin{aligned}
|(B k)(\eta)| & \leq \int_{\mathbb{R}^{d}} \sum_{\zeta \subset \eta, \zeta \neq \emptyset} \beta(x \mid \zeta)|k(\eta \cup x \backslash \zeta)| d x \\
& \leq \sum_{\zeta \subset \eta, \zeta \neq \emptyset}\|k\|_{\alpha^{\prime}}(|\eta|-|\zeta|+1) ! e^{-\alpha^{\prime}|\eta|} e^{(|\zeta|-1) \alpha^{\prime}}\left(\int_{\mathbb{R}^{d}} \beta(x \mid \zeta) d x\right) \\
& \leq|\eta|\|k\|_{\alpha^{\prime}} \bar{\varphi}|\eta| ! e^{-\alpha^{\prime}|\eta|}\left(\sum_{i=1}^{|\eta|} \frac{e^{(i-1) \alpha^{\prime}}}{i !}\right) \\
& \leq|\eta|\|k\|_{\alpha^{\prime}} \bar{\varphi}|\eta| ! e^{-\alpha^{\prime}|\eta|} H\left(\alpha^{\prime}\right),
\end{aligned}
$$

where we have also used that

$$
\sum_{i=1}^{|\eta|} \frac{e^{(i-1) \alpha^{\prime}}}{i !} \leq \sum_{i=1}^{\infty} \frac{e^{(i-1) \alpha^{\prime}}}{i !}=\frac{e^{e^{\alpha^{\prime}}}-1}{e^{\alpha^{\prime}}}=: H\left(\alpha^{\prime}\right) .
$$

Employing these estimates for calculating $\|A k\|_{\alpha}$ and $\|B k\|_{\alpha}$, we readily obtain (3.7).

By a classical solution of the problem (2.9), in a given $\mathcal{K}_{\alpha}$ and on the time interval $[0, T)$, we mean a continuous map $[0, T) \ni t \mapsto k_{t} \in \mathcal{D}_{\alpha}\left(L^{\Delta}\right)$ which is continuously differentiable in $\mathcal{K}_{\alpha}$ on $[0, T)$ and such that both equations in (2.9) are satisfied. Our main result is then given in the following statement.

Theorem 3.1. Let $\alpha_{0}$ and $\alpha_{*}$ be any real numbers and $\alpha_{0}>\alpha_{*}$. Then the problem (2.9) with $L^{\Delta}$ as in (3.2) and (3.3) for $k_{0} \in \mathcal{K}_{\alpha_{0}}$ has a unique classical solution $k_{t}$ in $\mathcal{K}_{\alpha_{*}}$ on the time interval $\left[0, T\left(\alpha_{*}\right)\right)$, where

$$
T\left(\alpha_{*}\right)=\frac{\alpha_{0}-\alpha_{*}}{\bar{\varphi} H\left(\alpha_{0}\right)} .
$$

Proof. We use a modification of the Ovcyannikov method, similar to that used in [6]. The estimates obtained above for $\|A k\|_{\alpha}$ and $\|B k\|_{\alpha}$ can also be used to define the corresponding bounded linear operators acting from $\mathcal{K}_{\alpha^{\prime}}$ to $\mathcal{K}_{\alpha}, \alpha^{\prime}>\alpha$. Let $\|\cdot\|_{\alpha \alpha^{\prime}}$ denote the operator norm. By means of the inequality

$$
|\eta| e^{-a|\eta|} \leq \frac{1}{e a}, \text { for } a>0 \text { and } \eta \in \Gamma_{0},
$$

we then get

$$
\|A\|_{\alpha \alpha^{\prime}} \leq \frac{\bar{\beta}}{e\left(\alpha^{\prime}-\alpha\right)}, \quad\|B\|_{\alpha \alpha^{\prime}} \leq \frac{\bar{\varphi} H\left(\alpha^{\prime}\right)}{e\left(\alpha^{\prime}-\alpha\right)} .
$$

Next, for $t>0$ and the same $\alpha, \alpha^{\prime}$, let us define the operator

$$
\mathcal{K}_{\alpha^{\prime}} \ni k \mapsto \Psi_{\alpha \alpha^{\prime}}(t) k \in \mathcal{K}_{\alpha},
$$

where

$$
\left(\Psi_{\alpha \alpha^{\prime}}(t) k\right)(\eta)=\exp (-t E(\eta)) k(\eta) .
$$


Clearly,

$$
\Psi_{\alpha \alpha^{\prime}}(t) \Psi_{\alpha^{\prime} \alpha^{\prime \prime}}(s)=\Psi_{\alpha \alpha^{\prime \prime}}(t+s),
$$

holding for all $t, s>0$ and $\alpha^{\prime \prime}>\alpha^{\prime}>\alpha$. Also

$$
\left\|\Psi_{\alpha \alpha^{\prime}}(t)\right\|_{\alpha \alpha^{\prime}} \leq 1, \quad t>0 .
$$

For $t=0$, (3.11) turns into the embedding operator $I_{\alpha \alpha^{\prime}}: \mathcal{K}_{\alpha^{\prime}} \rightarrow \mathcal{K}_{\alpha}$. Note that for each $t \geq 0$, the multiplication operator (3.12) can be defined as a bounded operator acting in the same space $\mathcal{K}_{\alpha}$. We define as in (3.11) to secure the continuity of the map $[0,+\infty) \ni t \mapsto \Psi_{\alpha \alpha^{\prime}} k \in \mathcal{K}_{\alpha}$ for each $k \in \mathcal{K}_{\alpha^{\prime}}$. Indeed, by (3.10) we get, cf. (3.13),

$$
\left\|\Psi_{\alpha \alpha^{\prime}}(t)-I_{\alpha \alpha^{\prime}}\right\|_{\alpha \alpha^{\prime}} \leq t\|A\|_{\alpha \alpha^{\prime}} \rightarrow 0, \quad \text { as } t \rightarrow 0 .
$$

Let $\alpha_{0}$ and $\alpha_{*}$ be as in the statement. For $t<T\left(\alpha_{*}\right)$, we pick $q>1$ such that also $q t<T\left(\alpha_{*}\right)$. For this $q$ and some $n \in \mathbb{N}$, we introduce the following partition of the interval $\left[\alpha_{*}, \alpha_{0}\right]$ :

$$
\begin{gathered}
\alpha_{2 p}=\alpha_{0}-p \frac{(q-1)\left(\alpha_{0}-\alpha_{*}\right)}{q(n+1)}-p \frac{\alpha_{0}-\alpha_{*}}{q n}, \\
\alpha_{2 p+1}=\alpha_{0}-(p+1) \frac{(q-1)\left(\alpha_{0}-\alpha_{*}\right)}{q(n+1)}-p \frac{\alpha_{0}-\alpha_{*}}{q n},
\end{gathered}
$$

where $p=0,1,2, \ldots, n$. Note that $\alpha_{2 n+1}=\alpha_{*}$. Let

$$
B_{n-p+1}: \mathcal{K}_{\alpha_{2 p-1}} \rightarrow \mathcal{K}_{\alpha_{2 p}}, \quad p=1,2, \ldots, n,
$$

act as defined in (3.6). Then the norm $\left\|B_{n-p+1}\right\|_{\alpha_{2 p} \alpha_{2 p-1}}$ can be estimated as in (3.10), which yields, see (3.14),

$$
\left\|B_{n-p+1}\right\|_{\alpha_{2 p} \alpha_{2 p-1}} \leq \frac{q n}{e T\left(\alpha_{*}\right)} .
$$

For each $m \in \mathbb{N}$, we then set

$$
\begin{aligned}
k_{t, m} & =\Psi_{\alpha_{*} \alpha_{0}}(t) k_{0} \\
& +\sum_{n=1}^{m} \int_{0}^{t} \int_{0}^{t_{1}} \ldots \int_{0}^{t_{n-1}} \Psi_{\alpha_{2 n+1} \alpha_{2 n}}\left(t-t_{1}\right) B_{1} \Psi_{\alpha_{2 n-1} \alpha_{2 n-2}}\left(t_{1}-t_{2}\right) B_{2} \ldots \\
& \times \Psi_{\alpha_{3} \alpha_{2}}\left(t_{n-1}-t_{n}\right) B_{n} \Psi_{\alpha_{1} \alpha_{0}}\left(t_{n}\right) k_{0} d t_{n} \ldots d t_{1} .
\end{aligned}
$$

By direct calculation we get that

$$
\frac{d}{d t} k_{t, m}=A k_{t, m}+B k_{t, m-1}
$$

where $A: \mathcal{D}_{\alpha_{*}}(A) \rightarrow \mathcal{K}_{\alpha_{*}}$ and $B: \mathcal{K}_{\alpha_{2 m-1}} \rightarrow \mathcal{K}_{\alpha_{*}}$. Note that

$$
\begin{aligned}
k_{t, n}-k_{t, n-1}= & \int_{0}^{t} \int_{0}^{t_{1}} \ldots \int_{0}^{t_{n-1}} \Psi_{\alpha_{2 n+1} \alpha_{2 n}}\left(t-t_{1}\right) B_{1} \Psi_{\alpha_{2 n-1} \alpha_{2 n-2}}\left(t_{1}-t_{2}\right) B_{2} \ldots \\
& \times \Psi_{\alpha_{3} \alpha_{2}}\left(t_{n-1}-t_{n}\right) B_{n} \Psi_{\alpha_{1} \alpha_{0}}\left(t_{n}\right) k_{0} d t_{n} \ldots d t_{1}
\end{aligned}
$$


which yields by (3.15)

$$
\left\|k_{t, n}-k_{t, n-1}\right\|_{\alpha_{*}} \leq \frac{1}{n !}\left(\frac{n}{e}\right)^{n}\left(\frac{q t}{T\left(\alpha_{*}\right)}\right)^{n}\left\|k_{0}\right\|_{\alpha_{0}} .
$$

The operators under the integrals in (3.16) are continuous (as the products of bounded operators), so $k_{t, n} \in \mathcal{K}_{\alpha_{*}}$ is continuous on $t \in\left[0, T\left(\alpha_{*}\right)\right)$. In view of (3.18), $\left\{k_{s, n}\right\}_{n \in \mathbb{N}_{0}}$ is a Cauchy sequence, uniformly in $s \in[0, t]$. Let $k_{s} \in \mathcal{K}_{\alpha_{*}}$ be the limit of this sequence, which then is a continuous function of $s \in\left[0, T\left(\alpha_{*}\right)\right)$. By repeating the above arguments one shows that the same is true in $\mathcal{K}_{\alpha_{*}+\epsilon} \hookrightarrow \mathcal{K}_{\alpha_{*}}$ for small enough $\epsilon>0$. Hence $k_{s} \in \mathcal{D}_{\alpha_{*}}\left(L^{\Delta}\right)$ for all $s \in\left[0, T\left(\alpha_{*}\right)\right)$. Next, by (3.10) and (3.18), $\left\{d k_{s, n} / d s\right\}_{n \in \mathbb{N}_{0}}$ is also a Cauchy sequence for $s \in[0, t]$ and $d k_{s, n} / d s \rightarrow d k_{s} / d s$ for $n \rightarrow \infty$. Hence, $k_{s}$ is the classical solution on $\left[0, T\left(\alpha_{*}\right)\right)$.

Now we show the uniqueness stated. Assume that $u_{t}$ and $v_{t}$ are two solution of (2.9) with (3.2). Then $w_{t}:=u_{t}-v_{t}$ satisfies (2.9) with the zero initial condition. For each $\tilde{\alpha}<\alpha_{*}$, the embedding $I_{\tilde{\alpha} \alpha_{*}}$ is continuous. Hence $w_{t}$ solves $(2.9)$ also in $\mathcal{K}_{\tilde{\alpha}}$. Then it can be written in the form

$$
w_{t}=\int_{0}^{t} \Psi_{\tilde{\alpha} \alpha}(t-s) B w_{s} d s,
$$

for some $\alpha \in\left(\tilde{\alpha}, \alpha_{*}\right)$. Here $w_{s}$ lies in $\mathcal{K}_{\alpha_{*}}$ and $B$ acts from $\mathcal{K}_{\alpha_{*}}$ to $\mathcal{K}_{\alpha}$. Now for a given $n>1$, we split $\left[\tilde{\alpha}, \alpha_{*}\right]$ similarly as above, i.e., set $\epsilon=\left(\alpha_{*}-\tilde{\alpha}\right) / 2 n$ and

$$
\alpha_{p}=\alpha_{*}-p \epsilon, \quad p=0, \ldots, 2 n .
$$

Then we reiterate (3.19) $n$ times and obtain

$$
\begin{aligned}
w_{t}= & \int_{0}^{t} \int_{0}^{t_{1}} \ldots \int_{0}^{t_{n-1}} \Psi_{\alpha_{2 n} \alpha_{2 n-1}}\left(t-t_{1}\right) B_{1} \Psi_{\alpha_{2 n-2} \alpha_{2 n-3}}\left(t_{1}-t_{2}\right) B_{2} \ldots \\
& \times \Psi_{\alpha_{2} \alpha_{1}}\left(t_{n-1}-t_{n}\right) B_{n} w_{t_{n}} d t_{n} \ldots d t_{1},
\end{aligned}
$$

where $w_{t_{n}}$ lies in $\mathcal{K}_{\alpha_{*}}$ and $B_{k}$ acts from $\mathcal{K}_{\alpha_{2 n-2 k}}$ to $\mathcal{K}_{\alpha_{2 n-2 k+1}}$, which yields

$$
\left\|B_{k}\right\|_{\alpha_{2 n-2 k+1} \alpha_{2 n-2 k}} \leq \frac{2 n \bar{\varphi} H\left(\alpha_{*}\right)}{e\left(\alpha_{*}-\tilde{\alpha}\right)}
$$

Hence,

$$
\left\|w_{t}\right\|_{\tilde{\alpha}} \leq \frac{1}{n !}\left(\frac{n}{e}\right)^{n}\left(2 t \frac{\bar{\varphi} H\left(\alpha_{*}\right)}{\alpha_{*}-\tilde{\alpha}}\right)^{n} \sup _{s \in[0, t]}\left\|w_{s}\right\|_{\tilde{\alpha}},
$$

where the latter supremum is finite as $w_{s}$ is continuous. Since $n$ is arbitrary, this means that $\left\|w_{t}\right\|_{\tilde{\alpha}}=0$ for

$$
t<\frac{\alpha_{*}-\tilde{\alpha}}{2 \bar{\varphi} H\left(\alpha_{*}\right)} .
$$

Then also $\left\|w_{t}\right\|_{\alpha_{*}}=0$. To extend this to the whole range of $t$ mentioned in the theorem one repeats the above construction due times. 
Acknowledgement. The research for this work was made possible thanks to the support given to the author during her stay at Bielefeld University in the framework of the joint Polish-German project No 57154469 "Dynamics of Large Systems of Interacting Entities" supported by the DAAD.

\section{REFERENCES}

[1] Albeverio, S., Kondratiev, Y., Röckner, M., Analysis and geometry on configuration spaces. J. Funct. Anal. 154 (1998), 444-500.

[2] Bogoliubov, N., Problems of a dynamical theory in statistical physics, in Studies in Statistical Mechanics, Vol. I (1962), 1-118.

[3] Boulanouar, M., The asymptotic behavior of a structured cell population, J. Evol. Equ. 11 (3) (2011), 531-552.

[4] Finkelshtein, D., Around Ovsyannikov's method, Methods Funct. Anal. Topology 21 (2) (2015), 134-150.

[5] Finkelshtein, D., Kondratiev, Y., Kozitsky, Y., Glauber dynamics in continuum: A constructive approach to evolution of states, Discrete Contin. Dyn. Syst. 33 (4) (2013), 1431-1450.

[6] Finkelshtein, D., Kondratiev, Y., Kozitsky, Y., Kutoviy, O., The statistical dynamics of a spatial logistic model and the related kinetic equation, Math. Models Methods Appl. Sci. 25 (2) (2015), 343-370.

[7] Finkelshtein, D., Kondratiev, Y., Kutoviy, O., Individual based model with competition in spatial ecology, SIAM J. Math. Anal. 41 (2009), 297-317.

[8] Finkelshtein, D., Kondratiev, Y., Oliveira, M. J., Markov evolution and hierarchical equations in the continuum. I: One-component systems, J. Evol. Equ. 9 (2009), 197233.

[9] Garcia, N. L., Kurtz, T. G., Spatial birth and death processes as solutions of stochastic equations, ALEA Lat. Am. J. Probab. Math. Stat. 1 (2006), 281-303.

[10] Kondratiev, Y., Kuna, T., Harmonic analysis on configuration space. I. General theory, Infin. Dimens. Anal. Quantum Probab. Relat. Top. 5 (2002), 201-233.

[11] Kondratiev, Y., Kuna, T., Oliveira, M. J., Holomorphic Bogoliubov functionals for interacting particle systems in continuum, J. Funct. Anal. 238 (2006), 375-404.

[12] Kondratiev, Y., Kutoviy, O., On the metrical properties of the configuration space, Math. Nachr. 279 (2006), 774-783.

[13] Kondratiev, Y., Kutoviy, O., Pirogov, S., Correlation functions and invariant measures in continuous contact model, Infin. Dimens. Anal. Quantum Probab. Relat. Top. 11 (2008), 231-258.

[14] Kozitsky, Y., Dynamics of spatial logistic model: finite systems, in Banasiak, J., Bobrowski, A., Lachowicz, M. (eds.) Semigroups of Operators - Theory and Applications, Będlewo, Poland, October 2013. Springer Proceedings in Mathematics \& Statistics 113, 2015, 197-211.

[15] Lebowitz, J. L., Rubinow, S. I., A theory for the age and generation time distribution of a microbial population, J. Math. Biol. 1 (1974), 17-36.

[16] Neuhauser, C., Mathematical challenges in spatial ecology, Notices Amer. Math. Soc. 48 (11) (2001), 1304-1314.

[17] Shanthidevi, C. N., Matsumoto, T., Oharu, S., Nonlinear semigroup approach to age structured proliferating cell population with inherited cycle length, Nonlinear Anal. Real World Appl. 9 (5) (2008), 1905-1917.

[18] Tréves, F., Ovcyannikov Theorem and Hyperdifferential Operators, Instituto de Matemática Pura e Aplicada, Conselho Nacional de Pesquisas, Rio de Janeiro, 1968. 
Agnieszka Tanaś

Institute of Mathematics

Maria Curie-Skłodowska University

pl. M. Curie-Skłodowskiej 1

20-031 Lublin

Poland

e-mail: agnieszka.puchacz@interia.eu

Received September 14, 2015 